\title{
The limits of viability in preterm infants, a growing ethical dilemma
}

\author{
Without ethics, everything happens as \\ if we were all five billion passengers on a \\ big machinery that nobody handles, and it's \\ going faster and faster, but we don't know \\ where. Jacques Cousteau
}

The remarkable advances in neonatal care of extremely preterm (EP) infants are increasingly changing the limits of viability towards progressively younger gestational ages at birth. In my opinion, this tendency places us in a difficult position where the potential benefits of survival are less significant than the long-term outcomes. Such behavior may be the consequence of the erroneous belief that medicine could achieve any and all accomplishments, even if they are not achievable due to the multiple biological hurdles faced by it. Unfortunately, when confronted with this situation, the fact that every medical practice should, above all, be ethical is most likely disregarded. In the absence of ethics, whatever we do is unfounded.

The limits of viability have reduced gradually over the last decades, from 28 weeks approximately 30 years ago to 26 weeks, then to 24 weeks, and now to 22 weeks. As these changes took place, mortality and morbidity in preterm infants reduced progressively, resulting in new and complex situations, not only for physicians, but also for parents and their families.

Some of the aspects that hinder the approach to EP infants include the multiple difficulties to establish an adequate definition of when treatment is fruitless and futile. This is mostly due to the uncertainty regarding the level of immaturity, severe morbidity, and future outcomes. I believe this situation is now more common in medicine because many assume that everything will be solved with technology; therefore, physicians fail to apply the concept of uncertainty.

It is evident that neonatologists deal with extremely complex situations when deciding on whether to start, continue or interrupt vital support in infants born at the limit of viability. The rule is to provide the greatest extent of care, at least in the first days; then the dilemma escalates if progress is not favorable and the infant's life depends on the implemented care measures, because suppressing these would result in his/her death. At the margins of viability, the interaction between physicians and families is highly challenging but it is also an opportunity to help parents in such a distressing and stressful situation.
However, this practice varies noticeably among health care providers. A group may agree on saving the infant's life "at all costs" and maintaining support, regardless of the future consequences on neurodevelopment and the parents' will. This leads to therapeutic obstinacy because, most likely, the outcomes of EP infants will not be sufficient to achieve an adequate clinical course. If health care providers insist on deciding not to perform a careful analysis of the situation, disproportionate treatments will prevail. In this way, both parents and physicians become the hostages of modern medicine, thus perpetuating inadequate therapeutics.

Another group of neonatologists will have a completely different attitude and maintain an open and compassionate relationship with the parents since the beginning, even before birth, and will explain the complications of their infant's treatment. It is frequent -and understandablethat parents have hopes at first and encourage the continuity of care, but as days go by and no recovery is seen, they realize treatment is futile and gradually change their mind. At a certain point, they usually state that they do not want to continue with treatment. Based on ethical principles, the group of health care providers will treat parents as their partners and collaborate with them by providing help, understanding, empathy, and an honest communication..$^{1-4}$

In addition, it is worth mentioning that it is usual to use gestational age as the only parameter in the long-term course, which is a serious mistake that has historically endured, and still persists. The aspects based on social variables have been gaining in importance. Doyle et al., ${ }^{5}$ conducted a study to assess the relevance of social and biological aspects in the long-term course in a group of preterm infants born with a birth weight of less than $1000 \mathrm{~g}$. They observed that social variables such as growing up in a multilingual household, having a low socioeconomic status, and a lower level of maternal education had a negative effect and were more relevant in late outcomes. Perinatal biological outcome measures showed important adverse associations, mainly in relation to cognitive and learning outcomes.

However, the great complexity in relation to the margins of viability to make decisions makes it necessary to consider, besides gestational age and social aspects, birth weight, sex, indication of antenatal corticosteroids, need of advanced resuscitation at birth, and severe morbidity in the first days of life. Each of these aspects should be 
taken into consideration because they may affect the infant's clinical course. Tyson et al. ${ }^{6}$ analyzed a large cohort of preterm infants born between 22 and 25 weeks of gestation and observed that those exposed to antenatal corticosteroids, of female sex, born to a singleton pregnancy, and with a higher birth weight (every $100 \mathrm{~g}$ of increment) had a lower risk for death and fewer severe sequelae on neurodevelopment. The likelihood of a favorable prognosis in the presence of these four variables served as a better estimator than considering only gestational age. Other studies found that the administration of antenatal corticosteroids alone reduced mortality and longterm disabilities. ${ }^{7,8}$

It is critical to bear in mind that these data stress the need to know other factors that may be part of the actions taken instead of making hasty decisions based exclusively on gestational age.

In relation to published studies that analyzed long-term follow-up of EP infants, it is worth noting the one by Marlow et al., ${ }^{9}$ who assessed a large cohort of preterm infants born between 22 and 25 weeks of gestation at 6 years old. They found a survival rate without disability at 6 years old of $0 \%, 1 \%, 3 \%$, and $8 \%$ among those born at $22,23,24$, and 25 weeks of gestation, respectively. Although that study was based exclusively on gestational age and may have been biased and present limitations, results were truly alarming. A recent systematic review assessed the cognitive outcomes of children who were born extremely preterm and at less than 32 weeks of gestation between 1990 and $2008 .{ }^{10} \mathrm{EP}$ infants showed important intelligence deficits and the cognitive outcome did not show improvements between 1990 and 2008. These findings underscore that neonatal care of very small preterm infants after birth is still a major dilemma. In addition, it was observed that bronchopulmonary dysplasia was by far the main adverse outcome measure in cognitive disorders.

As a corollary of the great complexity of the limits of viability, it is necessary to emphasize the need of having ethics in mind at all times so as to maintain adequate behaviors. The beneficence and the non maleficence principles point out that physicians should do good and avoid doing harm. These principles operate in every human action and are undisputed, but in such complex situations, they are certainly difficult to implement. Uncertainty regarding the course of EP infants in the short and long term makes us incapable of establishing an accurate prognosis, at least in the first weeks of life. Many times, this prevents us from firmly asserting whether we are doing good or harm. Therefore, it is critical that we become aware of our actions when faced with this situation.
The hospitalization of EP infants will invariably be very prolonged and pose many highly challenging situations for parents. Their stress and distress will only be mitigated by our appropriate conduct. Every action carried out under ethical principles during this extended stay will have a good impact and will give us the satisfaction of helping our fellow human beings, which has always been one of the main goals of medicine.

José M. Ceriani Cernadas Editor

http: / / dx.doi.org/10.5546/aap.2018.eng.170

To cite: Ceriani Cernadas JM. The limits of viability in preterm infants, a growing ethical dilemma. Arch Argent Pediatr 2018;116(3):170-171.

\section{REFERENCES}

1. Sebastiani M,Ceriani Cernadas JM. Aspectos bioéticos en el cuidado de los recién nacidos extremadamente prematuros. Arch Argent Pediatr 2008; 106 (3):242-8.

2. Myers P, Andrews B, Meadow W. Opportunities and difficulties for counseling at the margins of viability. Semin Fetal Neonatal Med 2017;23(1):30-4.

3. Ruthford E, Ruthford M, Hudak ML. Parent-Physician Partnership at the Edge of Viability. Pediatrics. 2017; 139(4):e20163899.

4. Raju TNK, Mercer BM, Burchfield DJ, et al. Periviable birth: executive summary of a Joint Workshop by the Eunice Kennedy Shriver National Institute of Child Health and Human Development, Society for MaternalFetal Medicine, American Academy of Pediatrics, and American College of Obstetricians and Gynecologists. J Perinatol 2014; (34)5: 333-42.

5. Doyle LW, Cheong JLY, Burnett A, et al. Biological and Soc th Weight Adolescents. Pediatrics. 2015; 136(6):1513-20.

6. Tyson JE, Parikh NA, Langer J, et al. National Institute of Child Health and Human Development Neonatal Research Network. Intensive care for extreme prematurity-moving beyond gestational age. N Engl J Med. 2008; 358(16):1672-81.

7. Chawla S, Natarajan G, Shankaran S, et al. Association of Neurodevelopmental Outcomes and Neonatal Morbidities of Extremely Premature Infants With Differential Exposure to Antenatal Steroids. JAMA Pediatr. 2016;170(12):1164-72.

8. Carlo WA, McDonald SA, Fanaroff AA, et al; Eunice Kennedy Shriver National Institute of Child Health and Human Development Neonatal Research Network. Association of antenatal corticosteroids with mortality and neurodevelopmental outcomes among infants born at 22 to 25 weeks' gestation. JAMA. 2011;306(21): 2348-58.

9. Marlow N, Wolke D, Bracewell M, et al. Neurologic and development disability at six years after extremely preterm birth. N Engl J Med. 2005; 352(1):9-19.

10. Twilhaar ES, Wade RM, de Kieviet JF, et al. Cognitive Outcomes of Children Born Extremely or Very Preterm Since the 1990s and Associated Risk Factors A Metaanalysis and Meta-regression. JAMA Pediatr. doi:10.1001/ jamapediatrics.2017.5323 Published online February19, 2018. 